\title{
THE TURNPIKE RESULT FOR APPROXIMATE SOLUTIONS OF NONAUTONOMOUS VARIATIONAL PROBLEMS
}

\author{
ALEXANDER J. ZASLAVSKI \\ (Received 31 April 2004; revised 26 October 2004)
}

Communicated by A. M. Rubinov

\begin{abstract}
In this work we study the structure of approximate solutions of variational problems with continuous integrands $f:[0, \infty) \times \mathbb{R}^{n} \times \mathbb{R}^{n} \rightarrow \mathbb{R}^{1}$ which belong to a complete metric space of functions. The main result in this paper deals with the turnpike property of variational problems. To have this property means that the approximate solutions of the problems are determined mainly by the integrand, and are essentially independent of the choice of interval and endpoint conditions, except in regions close to the endpoints.
\end{abstract}

2000 Mathematics subject classification: primary 49J99, $54 \mathrm{E} 52$.

Keywords and phrases: Complete metric space, good function, integrand, turnpike property.

\section{Introduction and main results}

In this paper we analyse the structure of solutions of the variational problems

(P) $\int_{T_{1}}^{T_{2}} f\left(t, z(t), z^{\prime}(t)\right) d t \rightarrow \min , \quad\left\{\begin{array}{l}z\left(T_{1}\right)=x, z\left(T_{2}\right)=y, z:\left[T_{1}, T_{2}\right] \rightarrow \mathbb{R}^{n} \text { is } \\ \text { an absolutely continuous (a.c.) function, }\end{array}\right.$

where $T_{1} \geq 0, T_{2}>T_{1}, x, y \in \mathbb{R}^{n}$ and $f:[0, \infty) \times \mathbb{R}^{n} \times \mathbb{R}^{n} \rightarrow \mathbb{R}^{\prime}$ belongs to a space of integrands described below.

Let $T_{1} \geq 0, T_{2}>T_{1}, x, y \in \mathbb{R}^{n}, f:[0, \infty) \times \mathbb{R}^{n} \times \mathbb{R}^{n} \rightarrow \mathbb{R}^{1}$ be an integrand and let $\delta$ be a positive number. We say that an absolutely continuous (a.c.) function $u:\left[T_{1}, T_{2}\right] \rightarrow \mathbb{R}^{n}$ satisfying $u\left(T_{1}\right)=x, u\left(T_{2}\right)=y$ is a $\delta$-approximate solution of the problem $(\mathrm{P})$ if

$$
\int_{T_{1}}^{T_{2}} f\left(t, u(t), u^{\prime}(t)\right) d t \leq \int_{T_{1}}^{T_{2}} f\left(t, z(t), z^{\prime}(t)\right) d t+\delta
$$

(C) 2006 Australian Mathematical Society $1446-7887 / 06 \$ A 2.00+0.00$ 
for each a.c. function $z:\left[T_{1}, T_{2}\right] \rightarrow \mathbb{R}^{n}$ satisfying $z\left(T_{1}\right)=x, z\left(T_{2}\right)=y$.

The main results in this paper deal with the so-called turnpike property of the variational problems $(\mathrm{P})$. To have this property means, roughly speaking, that the approximate solutions of the problems (P) are determined mainly by the integrand (cost function), and are essentially independent of the choice of interval and endpoint conditions, except in regions close to the endpoints.

Turnpike properties are well known in mathematical economics. The term was first coined by Samuelson in 1948 (see [12]) where he showed that an efficient expanding economy would spend most of the time in the vicinity of a balanced equilibrium path (also called a von Neumann path). This property was further investigated for optimal trajectories of models of economic dynamics (see, for example, [2, 3, 5-11] and the references mentioned there). In control theory turnpike properties were studied in $[18,19]$ for linear control systems with convex integrands.

Denote by $|\cdot|$ the Euclidean norm in $\mathbb{R}^{n}$. Let $a>0$ be a positive constant and let $\psi:[0, \infty) \rightarrow[0, \infty)$ be an increasing function such that $\psi(t) \rightarrow+\infty$ as $t \rightarrow \infty$. Denote by $\mathscr{M}$ the set of all continuous functions $f:[0, \infty) \times \mathbb{R}^{n} \times \mathbb{R}^{n} \rightarrow \mathbb{R}^{1}$ which satisfy the following assumptions:

(A.i) The function $f$ is bounded on $[0, \infty) \times E$ for any bounded set $E \subset \mathbb{R}^{n} \times \mathbb{R}^{n}$. (A.ii) $f(t, x, u) \geq \max \{\psi(|x|), \psi(|u|)|u|\}-a$ for each $(t, x, u) \in[0, \infty) \times \mathbb{R}^{n} \times$ $\mathbb{R}^{n}$.

(A.iii) For each $M, \epsilon>0$, there exist $\Gamma, \delta>0$ such that

$$
\left|f\left(t, x_{1}, u\right)-f\left(t, x_{2}, u\right)\right| \leq \epsilon \max \left\{f\left(t, x_{1}, u\right), f\left(t, x_{2}, u\right)\right\}
$$

for each $t \in[0, \infty)$ and each $u, x_{1}, x_{2} \in \mathbb{R}^{n}$ which satisfy

$$
\left|x_{i}\right| \leq M, i=1,2,|u| \geq \Gamma, \quad\left|x_{1}-x_{2}\right| \leq \delta .
$$

(A.iv) For each $M, \epsilon>0$, there exists $\delta>0$ such that

$$
\left|f\left(t, x_{1}, u_{1}\right)-f\left(t, x_{2}, u_{2}\right)\right| \leq \epsilon
$$

for each $t \in[0, \infty)$ and each $u_{1}, u_{2}, x_{1}, x_{2} \in \mathbb{R}^{n}$ which satisfy

$$
\left|x_{i}\right|,\left|u_{i}\right| \leq M, i=1,2, \quad \max \left\{\left|x_{1}-x_{2}\right|,\left|u_{1}-u_{2}\right|\right\} \leq \delta .
$$

In $[16,17]$ we studied the subset of the set $\mathscr{M}$ which consists of all $f \in \mathscr{M}$ satisfying the following assumptions:

- for each $(t, x) \in[0, \infty) \times \mathbb{R}^{n}$ the function $f(t, x, \cdot): \mathbb{R}^{n} \rightarrow \mathbb{B}^{1}$ is convex;

- for each $M, \epsilon>0$ there exist $\Gamma, \delta>0$ such that

$$
\left|f\left(t, x_{1}, u_{1}\right)-f\left(t, x_{2}, u_{2}\right)\right| \leq \epsilon \max \left\{f\left(t, x_{1}, u_{1}\right), f\left(t, x_{2}, u_{2}\right)\right\}
$$


for each $t \in[0, \infty)$ and each $u_{1}, u_{2}, x_{1}, x_{2} \in \mathbb{R}^{n}$ which satisfy

$$
\left|x_{i}\right| \leq M,\left|u_{i}\right| \geq \Gamma, i=1,2, \quad \max \left\{\left|x_{1}-x_{2}\right|,\left|u_{1}-u_{2}\right|\right\} \leq \delta
$$

(see (A.iii)).

This subset will be denoted by $\mathscr{M}_{c o}$.

It is easy to show that an integrand $f=f(t, x, u) \in C^{1}\left([0, \infty) \times \mathbb{R}^{n} \times \mathbb{R}^{n}\right)$ belongs to $\mathscr{M}$ if $f$ satisfies assumption (A.ii), and if $\sup \{|f(t, 0,0)|: t \in[0, \infty)\}<\infty$ and also there exists an increasing function $\psi_{0}:[0, \infty) \rightarrow[0, \infty)$ such that

$$
\sup \left\{\left|\frac{\partial f(t, x, u)}{\partial x}\right|,\left|\frac{\partial f(t, x, u)}{\partial u}\right|\right\} \leq \psi_{0}(|x|)(1+\psi(|u|)|u|)
$$

for each $t \in[0, \infty)$ and each $x, u \in \mathbb{R}^{n}$.

For the set $\mathscr{M}$, we consider the uniformity which is determined by the following base:

$$
\begin{array}{r}
E(N, \epsilon, \lambda)=\{(f, g) \in \mathscr{M} \times \mathscr{M}:|f(t, x, u)-g(t, x, u)| \leq \epsilon \text { for each } \\
t \in[0, \infty) \text { and each } x, u \in \mathbb{R}^{n} \text { satisfying }|x|,|u| \leq N \\
\text { and }(|f(t, x, u)|+1)(|g(t, x, u)|+1)^{-1} \in\left[\lambda^{-1}, \lambda\right] \text { for each } \\
\\
\left.t \in[0, \infty) \text { and each } x, u \in \mathbb{R}^{n} \text { satisfying }|x| \leq N\right\},
\end{array}
$$

where $N>0, \epsilon>0, \lambda>1$.

Clearly, the space $\mathscr{M}$ with this uniformity is metrizable (by a metric $\rho_{w}$ ). It was established in [13, Proposition 2.2] that the metric space $\left(\mathscr{M}, \rho_{w}\right)$ is complete. Note that this uniformity was introduced in [16] for the subset $\mathscr{M}_{c o}$ of $\mathscr{M}$. The metric $\rho_{w}$ induces in $\mathscr{M}$ a topology.

We consider functionals of the form

$$
I^{f}\left(T_{1}, T_{2}, x\right)=\int_{T_{1}}^{T_{2}} f\left(t, x(t), x^{\prime}(t)\right) d t
$$

where $f \in \mathscr{M}, 0 \leq T_{1}<T_{2}<+\infty$ and $x:\left[T_{1}, T_{2}\right] \rightarrow \mathbb{R}^{n}$ is an a.c. function.

For $f \in \mathscr{M}, y, z \in \mathbb{R}^{n}$ and numbers $T_{1}, T_{2}$ satisfying $0 \leq T_{1}<T_{2}$ we set

$$
\begin{array}{r}
U^{f}\left(T_{1}, T_{2}, y, z\right)=\inf \left\{I^{f}\left(T_{1}, T_{2}, x\right) \mid x:\left[T_{1}, T_{2}\right] \rightarrow \mathbb{R}^{n}\right. \text { is an a.c. } \\
\text { function satisfying } \left.x\left(T_{1}\right)=y, x\left(T_{2}\right)=z\right\} .
\end{array}
$$

It is easy to see that $-\infty<U^{f}\left(T_{1}, T_{2}, y, z\right)<+\infty$ for each $f \in \mathfrak{M}$, each $y, z \in \mathbb{R}^{n}$ and all numbers $T_{1}, T_{2}$ satisfying $0 \leq T_{1}<T_{2}$.

Let $f \in \mathscr{M}$. A locally absolutely continuous (a.c.) function $x:[0, \infty) \rightarrow \mathbb{R}^{n}$ is called an $(f)$-good function if for any a.c. function $y:[0, \infty) \rightarrow \mathbb{R}^{n}$ there is a number $M_{y}$ such that

$$
I^{f}(0, T, y) \geq M_{y}+I^{f}(0, T, x) \text { for each } T \in(0, \infty) .
$$

In [14, Proposition 1.1] we proved the following result. 
Proposition 1.1. Let $f \in \mathscr{M}$ and let $x:[0, \infty) \rightarrow \mathbb{R}^{n}$ be a bounded a.c. function. Then the function $x$ is $(f)$-good if and only if there is $M>0$ such that

$$
I^{f}(0, T, x) \leq U^{f}(0, T, x(0), x(T))+M \text { for any } T>0 .
$$

The next result will be proved in Section 3 .

PROPOSITION 1.2. Let $f \in \mathscr{M}$ and let for each $(t, x) \in[0, \infty) \times \mathbb{R}^{n}$ the function $f(t, x, \cdot): \mathbb{R}^{n} \rightarrow \mathbb{R}^{\prime}$ be convex. Then for each $z \in \mathbb{R}^{n}$ there is a bounded $(f)$-good function $Z:[0, \infty) \rightarrow \mathbb{R}^{n}$ such that $Z(0)=z$ and that for each $T>0$,

$$
I^{f}(0, T, Z)=U^{f}(0, T, Z(0), Z(T)) .
$$

Let $f \in \mathscr{M}$. We say that $f$ has the strong turnpike property, or briefly (STP), if there exists a bounded a.c. function $X_{f}:[0, \infty) \rightarrow \mathbb{R}^{n}$ which satisfies the following condition: For each $K, \epsilon>0$ there exist constants $\delta, L>0$ such that for each $T_{1} \geq 0, T_{2} \geq T_{1}+2 L$ and each a.c. function $v:\left[T_{1}, T_{2}\right] \stackrel{\square}{\rightarrow} \mathbb{R}^{n}$ which satisfies $\left|v\left(T_{1}\right)\right|,\left|v\left(T_{2}\right)\right| \leq K$ and $I^{f}\left(T_{1}, T_{2}, v\right) \leq U^{f}\left(T_{1}, T_{2}, v\left(T_{1}\right), v\left(T_{2}\right)\right)+\delta$

(i) there are $\tau_{1} \in\left[T_{1}, T_{1}+L\right]$ and $\tau_{2} \in\left[T_{2}-L, T_{2}\right]$ for which

$$
\left|v(t)-X_{f}(t)\right| \leq \epsilon, \quad t \in\left[\tau_{1}, \tau_{2}\right]
$$

(ii) if $\left|v\left(T_{1}\right)-X_{f}\left(T_{1}\right)\right| \leq \delta$, then $\tau_{1}=T_{1}$ and if $\left|v\left(T_{2}\right)-X_{f}\left(T_{2}\right)\right| \leq \delta$, then $\tau_{2}=T_{2}$.

The function $X_{f}$ is called the turnpike of $f$.

If the integrand $f$ has the strong turnpike property, then the solutions of variational problems with $f$ are essentially independent of the choice of time interval and values at the endpoints except in regions close to the endpoints of the time interval. If a point $t$ does not belong to these regions, then the value of a solution at $t$ is closed to a trajectory ("turnpike") which is defined on the infinite time interval and depends only on $f$. This phenomenon has the following interpretation. If one wish to reach a point $A$ from a point $B$ by a car in an optimal way, then one should turn to a turnpike, spend most of time on it and then leave the turnpike to reach the required point.

If in the definition above condition (ii) is not assumed, then we say that the integrand $f$ has the turnpike property $[14,15,17]$.

In the sequel we use the following definition [4].

Let $f \in \mathscr{M}$. We say that an a.c. function $x:[0, \infty) \rightarrow \mathbb{R}^{n}$ is $(f)$-overtaking optimal if for each a.c. function $y:[0, \infty) \rightarrow \mathbb{R}^{n}$ satisfying $y(0)=x(0)$,

$$
\limsup _{r \rightarrow \Upsilon}\left[I^{f}(0, T, x)-I^{f}(0, T, y)\right] \leq 0 \text {. }
$$


Assume that $f \in \mathscr{M}$ and $X:[0, \infty) \rightarrow \mathbb{R}^{n}$ is a bounded a.c. function. How to verify if the integrand $f$ has (STP) and $X$ is its turnpike? In this paper we introduce three properties (P1), (P2) and (P3) and show that $f$ has (STP) if and only if $f$ possesses properties (P1), (P2) and (P3). Property (P1) means that all $(f)$-good functions have the same asymptotic behavior while property $(\mathrm{P} 2)$ means that $X$ is a unique $(f)$-overtaking optimal function whose value at zero is $X(0)$. Property (P3) means that if an a.c. function $v:[0, T] \rightarrow \mathbb{R}^{n}$ is an approximate solution and $T$ is large enough, then there is $\tau \in[0, T]$ such that $v(\tau)$ is close to $X(\tau)$. In [14] we establish that $f$ has the turnpike property if and only if $f$ possesses properties (P1) and (P3).

The next theorem is the main result of the paper.

THEOREM 1.3. Let $f \in \mathscr{M}$, for each $(t, x) \in[0, \infty) \times \mathbb{R}^{n}$ the function $f(t, x, \cdot)$ : $\mathbb{R}^{n} \rightarrow \mathbb{R}^{1}$ be convex and let $X_{f}:[0, \infty) \rightarrow \mathbb{R}^{n}$ be a bounded a.c. function. Then $f$ has the strong turnpike property with $X_{f}$ being the turnpike if and only if the following three properties hold:

(P1) For each pair of $(f)$-good functions $v_{1}, v_{2}:[0, \infty) \rightarrow \mathbb{R}^{n}$,

$$
\left|v_{1}(t)-v_{2}(t)\right| \rightarrow 0 \text { as } t \rightarrow \infty
$$

(P2) $X_{f}$ is an $(f)$-overtaking optimal function and if an $(f)$-overtaking optimal function $v:[0, \infty) \rightarrow \mathbb{R}^{n}$ satisfies $v(0)=X_{f}(0)$, then $v=X_{f}$.

(P3) For each $K, \epsilon>0$ there exist $\gamma, l>0$ such that for each $T \geq 0$ and each a.c. function $w:[T, T+l] \rightarrow \mathbb{B}^{n}$ which satisfies $|w(T)|,|w(T+l)| \leq K$ and $I^{f}(T, T+l, w) \leq U^{f}(T, T+l, w(T), w(T+l))+\gamma$ there is $\tau \in[T, T+l]$ for which $\left|X_{f}(\tau)-v(\tau)\right| \leq \epsilon$.

\section{Auxiliary results}

We have the following result (see Berkovitz [1]).

Proposition 2.1. Assume that $f \in \mathscr{M}$ and $f(t, x, \cdot): \mathbb{R}^{n} \rightarrow \mathbb{R}^{1}$ is a convex function for each $(t, x) \in \mathbb{R}^{n} \times[0, \infty)$. Then for each pair of numbers $T_{1}, T_{2}$ satisfying $0 \leq T_{1}<T_{2}$ and each $z_{1}, z_{2} \in \mathbb{R}^{n}$ there exists an a.c. function $x:\left[T_{1}, T_{2}\right] \rightarrow \mathbb{R}^{n}$ such that

$$
x\left(T_{i}\right)=z_{i}, \quad i=1,2, \quad I^{f}\left(T_{1}, T_{2}, x\right)=U^{f}\left(T_{1}, T_{2}, z_{1}, z_{2}\right) .
$$

In [13] we analyzed the properties of $(f)$-good functions and established the following results. 
PROPOSITION 2.2 ([13, Theorem 1.1]). For each $h \in \mathscr{M}$, each $\delta \in(0,1)$ and each $z \in \mathbb{B}^{n}$, there exists an $(h)$-good function $Z_{\delta}^{h}:[0, \infty) \rightarrow \mathbb{B}^{n}$ satisfying $Z_{\delta}^{h}(0)=z$ such that the following assertions hold:

(1) Let $f \in \mathscr{M}, \epsilon \in(0,1), z \in \mathbb{R}^{n}$ and let $y:[0, \infty) \rightarrow \mathbb{R}^{n}$ be an a.c. function. Then one of the following properties holds:

(i) $I^{f}(0, T, y)-I^{f}\left(0, T, Z_{\epsilon}^{f}\right) \rightarrow \infty$ as $T \rightarrow \infty$;

(ii) $\sup \left\{\left|I^{f}(0, T, y)-I^{f}\left(0, T, Z_{\epsilon}^{f}\right)\right|: T \in(0, \infty)\right\}<\infty$ and

$$
\sup \{|y(t)|: t \in[0, \infty)\}<\infty \text {. }
$$

(2) For each $f \in \mathscr{M}$ and each positive number $M$, there exist a neighborhood $U$ of $f$ in $\mathscr{M}$ and a number $Q>0$ such that $\sup \left\{\left|Z_{\epsilon}^{g}(t)\right|: t \in[0, \infty)\right\} \leq Q$ for each $g \in U$, each $\epsilon \in(0,1)$ and each $z \in \mathbb{R}^{n}$ satisfying $|z| \leq M$.

(3) For each $f \in \mathscr{M}$ and each positive number $M$, there exist a neighborhood $U$ of $f$ in $\mathscr{M}$ and a number $Q>0$ such that for each $g \in U$, each $z \in \mathbb{R}^{n}$ satisfying $|z| \leq M$, each $\epsilon \in(0,1)$, each $T_{1} \geq 0, T_{2}>T_{1}$ and each a.c. function $y:\left[T_{1}, T_{2}\right] \rightarrow \mathbb{R}^{n}$ satisfying $\left|y\left(T_{1}\right)\right| \leq M$ the following relation holds:

$$
I^{g}\left(T_{1}, T_{2}, Z_{\epsilon}^{g}\right) \leq I^{g}\left(T_{1}, T_{2}, y\right)+Q .
$$

(4) For each $f \in \mathscr{M}, \epsilon>0, z \in \mathbb{R}^{n}, T_{1} \geq 0$ and $T_{2}>T_{1}$,

$$
I^{f}\left(T_{1}, T_{2}, Z_{\epsilon}^{f}\right) \leq U^{f}\left(T_{1}, T_{2}, Z_{\epsilon}^{f}\left(T_{1}\right), Z_{\epsilon}^{f}\left(T_{2}\right)\right)+\epsilon .
$$

(5) For each $f \in \mathscr{M}, z \in \mathbb{R}^{n}$ and an integer $i \geq 0$,

$$
Z_{\epsilon_{1}}^{f}(i)=Z_{\epsilon_{2}}^{f}(i) \text { for each } \epsilon_{1}, \epsilon_{2} \in(0,1) .
$$

Proposition 2.2 is an extension of [16, Theorem 1.1] which was established for the

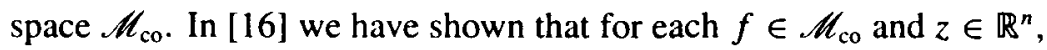

$$
Z_{\epsilon_{1}}^{f}=Z_{\epsilon_{2}}^{f} \text { for each } \epsilon_{1}, \epsilon_{2} \in(0,1)
$$

and

$$
U^{f}\left(T_{1}, T_{2}, Z_{\epsilon}^{f}\left(T_{1}\right), Z_{\epsilon}^{f}\left(T_{2}\right)\right)=I^{f}\left(T_{1}, T_{2}, Z_{\epsilon}^{f}\right)
$$

for each $T_{1} \geq 0, T_{2}>T_{1}$ and each $\epsilon \in(0,1)$.

Proposition 2.3 ([13, Proposition 2.6]). Let $f \in \mathscr{M}, 0<c_{1}<c_{2}<\infty$ and let $M, \epsilon>0$. Then there exists $\delta>0$ such that for each $T_{1}, T_{2} \geq 0$ satisfying $T_{2}-T_{1} \in\left[c_{1}, c_{2}\right]$ and each $y_{1}, y_{2}, z_{1}, z_{2} \in \mathbb{R}^{n}$ satisfying

$$
\left|y_{i}\right|,\left|z_{i}\right| \leq M, \quad i=1,2, \quad\left|y_{1}-y_{2}\right|,\left|z_{1}-z_{2}\right| \leq \delta
$$

the relation $\left|U^{f}\left(T_{1}, T_{2}, y_{1}, z_{1}\right)-U^{f}\left(T_{1}, T_{2}, y_{2}, z_{2}\right)\right| \leq \epsilon$ holds. 
PROPOSITION 2.4 ([13, Theorem 1.3]). Let $f \in \mathscr{M}$ and let $M_{1}, M_{2}, c$ be positive numbers. Then there exist a neighborhood $\mathscr{U}$ of $f$ in $\mathscr{M}$ and a number $S>0$ such that for each $g \in \mathscr{U}$, each $T_{1} \in[0, \infty)$ and each $T_{2} \in\left[T_{1}+c, \infty\right)$ the following property holds: For each $x, y \in \mathbb{R}^{n}$ satisfying $|x|,|y| \leq M_{1}$ and each a.c. function $v:\left[T_{1}, T_{2}\right] \rightarrow \mathbb{R}^{n}$ satisfying

$$
v\left(T_{1}\right)=x, \quad v\left(T_{2}\right)=y, \quad I^{g}\left(T_{1}, T_{2}, v\right) \leq U^{g}\left(T_{1}, T_{2}, x, y\right)+M_{2},
$$

inequality $|v(t)| \leq S$ is valid for $t \in\left[T_{1}, T_{2}\right]$.

PROPOSITION 2.5 ([13, Proposition 2.4]). Let $M_{1}, \epsilon>0,0<\tau_{0}<\tau_{1}$. Then there exists $\delta>0$ such that for each $f \in \mathscr{M}$, each $T_{1} \in[0, \infty), T_{2} \in\left[T_{1}+\tau_{0}, T_{1}+\tau_{1}\right]$, each a.c. function $x:\left[T_{1}, T_{2}\right] \rightarrow \mathbb{R}^{n}$ satisfying $I^{f}\left(T_{1}, T_{2}, x\right) \leq M_{1}$ and each $t_{1}, t_{2} \in\left[T_{1}, T_{2}\right]$ which satisfy $\left|t_{2}-t_{1}\right| \leq \delta$, relation $\left|x\left(t_{1}\right)-x\left(t_{2}\right)\right| \leq \epsilon$ holds.

PROPOSITION 2.6 ([13, Proposition 2.5]). Let $f \in \mathscr{M}, 0<c_{1}<c_{2}<\infty$ and $c_{3}>0$. Then there exists a neighborhood $\mathscr{U}$ of $f$ in $\mathscr{M}$ such that the set

$$
\begin{gathered}
\left\{U^{g}\left(T_{1}, T_{2}, z_{1}, z_{2}\right): g \in \mathscr{U}, T_{1} \in[0, \infty), T_{2} \in\left[T_{1}+c_{1}, T_{1}+c_{2}\right],\right. \\
\left.z_{1}, z_{2} \in \mathbb{R}^{n},\left|z_{i}\right| \leq c_{3}, i=1,2\right\}
\end{gathered}
$$

is bounded.

PROPOSITION 2.7. Let $T_{1} \geq 0, T_{2}>T_{1}$ and let $v:\left[T_{1}, T_{2}\right] \rightarrow \mathbb{R}^{n}$ be a continuous function. Assume that for each $\tau_{1}, \tau_{2} \in\left(T_{1}, T_{2}\right)$ satisfying $\tau_{1}<\tau_{2}$ the restriction of $v$ to $\left[\tau_{1}, \tau_{2}\right]$ is an a.c. function and

$$
I^{f}\left(\tau_{1}, \tau_{2}, v\right)=U^{f}\left(\tau_{1}, \tau_{2}, v\left(\tau_{1}\right), v\left(\tau_{2}\right)\right) .
$$

Then the function $v:\left[T_{1}, T_{2}\right] \rightarrow \mathbb{R}^{n}$ is an a.c. function and

$$
I^{f}\left(T_{1}, T_{2}, v\right)=U^{f}\left(T_{1}, T_{2}, v\left(T_{1}\right), v\left(T_{2}\right)\right) .
$$

Proof. Choose

$$
M_{0}>\sup \left\{|v(t)|: t \in\left[T_{1}, T_{2}\right]\right\} .
$$

By (2.1), (2.3) and Proposition 2.6 the set

$$
\left\{I^{f}\left(\tau_{1}, \tau_{2}, v\right): \tau_{1}, \tau_{2} \in\left(T_{1}, T_{2}\right), \tau_{2}-\tau_{1} \in\left(0,\left(T_{2}-T_{1}\right) / 8\right)\right\}
$$

is bounded. It follows from this fact, (A.ii) and Fatou's lemma that the integral

$$
\int_{T_{1}}^{T_{2}} f\left(t, v(t), v^{\prime}(t)\right) d t
$$


is finite. Then (A.ii) implies that $v^{\prime} \in L^{1}\left(\left[T_{1}, T_{2}\right] ; \mathbb{R}^{n}\right)$ and $v:\left[T_{1}, T_{2}\right] \rightarrow \mathbb{R}^{n}$ is an a.c. function.

We show that (2.2) holds. Assume the contrary. Then there is an a.c. function $u:\left[T_{1}, T_{2}\right] \rightarrow \mathbb{R}^{n}$ such that

$$
u\left(T_{i}\right)=v\left(T_{i}\right), \quad i=1,2, \quad I^{f}\left(T_{1}, T_{2}, v\right)-I^{f}\left(T_{1}, T_{2}, u\right)>2 \Delta
$$

with $\Delta>0$.

It is not difficult to see that there is $\gamma \in\left(0,\left(T_{2}-T_{1}\right) / 8\right)$ such that:

$$
\begin{aligned}
& \left|I^{f}\left(s_{1}, s_{2}, v\right)\right| \leq \Delta / 64 \text { for each } s_{1}, s_{2} \in\left[T_{1}, T_{1}+\gamma\right] \text { satisfying } s_{2}>s_{1}, \\
& \left|I^{f}\left(s_{1}, s_{2}, v\right)\right| \leq \Delta / 64 \text { for each } s_{1}, s_{2} \in\left[T_{2}-\gamma, T_{2}\right] \text { satisfying } s_{2}>s_{1}, \\
& \left|I^{f}\left(s_{1}, s_{2}, u\right)\right| \leq \Delta / 64 \text { for each } s_{1}, s_{2} \in\left[T_{1}, T_{1}+\gamma\right] \text { satisfying } s_{2}>s_{1}, \\
& \left|I^{f}\left(s_{1}, s_{2}, u\right)\right| \leq \Delta / 64 \text { for each } s_{1}, s_{2} \in\left[T_{2}-\gamma, T_{2}\right] \text { satisfying } s_{2}>s_{1} .
\end{aligned}
$$

Choose a number

$$
M_{1}>\sup \left\{|v(t)|: t \in\left[T_{1}, T_{2}\right]\right\}+\sup \left\{|u(t)|: t \in\left[T_{1}, T_{2}\right]\right\}
$$

By Proposition 2.3 there is $\delta>0$ such that the following property holds: For each $t_{1} \geq 0, t_{2} \in\left[t_{1}+\gamma / 16, t_{1}+16 \gamma\right]$ and each $x_{1}, x_{2}, y_{1}, y_{2} \in \mathbb{R}^{n}$ satisfying

$$
\left|x_{i}\right|,\left|y_{i}\right| \leq M_{1}, \quad i=1,2, \quad\left|x_{i}-y_{i}\right| \leq \delta, \quad i=1,2,
$$

the inequality

$$
\left|U^{f}\left(t_{1}, t_{2}, x_{1}, x_{2}\right)-U^{f}\left(t_{1}, t_{2}, y_{1}, y_{2}\right)\right| \leq \Delta / 64
$$

is true.

Choose numbers $t_{1}, t_{2}$ such that

$$
\begin{gathered}
t_{1} \in\left(T_{1}, T_{1}+\gamma / 4\right], \quad t_{2} \in\left[T_{2}-\gamma / 4, T_{2}\right], \\
\left|v\left(T_{1}\right)-v\left(t_{1}\right)\right|,\left|u\left(T_{1}\right)-u\left(t_{1}\right)\right| \leq \delta / 4, \\
\left|v\left(T_{2}\right)-v\left(t_{2}\right)\right|,\left|u\left(T_{2}\right)-v\left(t_{2}\right)\right| \leq \delta / 4 .
\end{gathered}
$$

Relations (2.11) and (2.4) imply that, for $i=1,2$,

$$
\left|v\left(t_{i}\right)-u\left(t_{i}\right)\right| \leq\left|v\left(t_{i}\right)-v\left(T_{i}\right)\right|+\left|v\left(T_{i}\right)-u\left(T_{i}\right)\right|+\left|u\left(T_{i}\right)-u\left(t_{i}\right)\right| \leq \delta / 2 .
$$

Consider an a.c. function $\tilde{u}:\left[t_{1}, t_{2}\right] \rightarrow \mathbb{B}^{n}$ such that

$$
\tilde{u}(t)=u(t), t \in\left[T_{1}+\gamma, T_{2}-\gamma\right], \quad \tilde{u}\left(t_{i}\right)=v\left(t_{i}\right), i=1,2,
$$


and

$$
\begin{aligned}
& I^{f}\left(t_{1}, T_{1}+\gamma, \tilde{u}\right) \leq U^{f}\left(t_{1}, T_{1}+\gamma, v\left(t_{1}\right), u\left(T_{1}+\gamma\right)\right)+\Delta / 128 \\
& I^{f}\left(T_{2}-\gamma, t_{2}, \tilde{u}\right) \leq U^{f}\left(t_{2}, T_{2}-\gamma, u\left(T_{2}-\gamma\right), v\left(t_{2}\right)\right)+\Delta / 128 .
\end{aligned}
$$

It follows from (2.10), the choice of $\gamma$ (see (2.5), (2.6)) and (2.4) that

$$
\begin{aligned}
I^{f}\left(t_{1}, t_{2}, v\right)-I^{f}\left(t_{1}, t_{2}, u\right)= & I^{f}\left(T_{1}, T_{2}, v\right)-I^{f}\left(T_{1}, T_{2}, u\right)-I^{f}\left(T_{1}, t_{1}, v\right) \\
& -I^{f}\left(t_{2}, T_{2}, v\right)+I^{f}\left(T_{1}, t_{1}, u\right)+I^{f}\left(t_{2}, T_{2}, u\right) \\
\geq & 2 \Delta-4(\Delta / 64)>3 \Delta / 2 .
\end{aligned}
$$

In view of (2.13) and (2.1)

$$
\begin{aligned}
I^{f}\left(t_{1}, t_{2}, \tilde{u}\right)-I^{f}\left(t_{1}, t_{2}, u\right)= & I^{f}\left(t_{1}, T_{1}+\gamma, \tilde{u}\right)-I^{f}\left(t_{1}, T_{1}+\gamma, u\right) \\
& +I^{f}\left(T_{2}-\gamma, t_{2}, \tilde{u}\right)-I^{f}\left(T_{2}-\gamma, t_{2}, u\right) \\
\leq & U^{f}\left(t_{1}, T_{1}+\gamma, v\left(t_{1}\right), u\left(T_{1}+\gamma\right)\right)+\Delta / 128 \\
& -U^{f}\left(t_{1}, T_{1}+\gamma, u\left(t_{1}\right), u\left(T_{1}+\gamma\right)\right) \\
& +U^{f}\left(t_{2}, T_{2}-\gamma, u\left(T_{2}-\gamma\right), v\left(t_{2}\right)\right)+\Delta / 128 \\
& -U^{f}\left(T_{2}-\gamma, t_{2}, u\left(T_{2}-\gamma\right), u\left(t_{2}\right)\right) .
\end{aligned}
$$

It follows from (2.12), (2.7), (2.10) and the choice of $\delta$ (see (2.8), (2.9)) that

$$
\begin{aligned}
& \left|U^{f}\left(t_{1}, T_{1}+\gamma, u\left(t_{1}\right), u\left(T_{1}+\gamma\right)\right)-U^{f}\left(t_{1}, T_{1}+\gamma, v\left(t_{1}\right), u\left(T_{1}+\gamma\right)\right)\right| \leq \Delta / 64, \\
& \left|U^{f}\left(T_{2}-\gamma, t_{2}, u\left(T_{2}-\gamma\right), u\left(t_{2}\right)\right)-U^{f}\left(T_{2}-\gamma, t_{2}, u\left(T_{2}-\gamma\right), v\left(t_{2}\right)\right)\right| \leq \Delta / 64 .
\end{aligned}
$$

Relations (2.15) and (2.16) imply that

$$
I^{f}\left(t_{1}, t_{2}, \tilde{u}\right)-I^{f}\left(t_{1}, t_{2}, u\right) \leq \Delta / 64+\Delta / 64+\Delta / 64<\Delta / 16 .
$$

By (2.17) and (2.14),

$$
\begin{aligned}
& I^{f}\left(t_{1}, t_{2}, v\right)-I^{f}\left(t_{1}, t_{2}, \tilde{u}\right) \\
& \quad=I^{f}\left(t_{1}, t_{2}, v\right)-I^{f}\left(t_{1}, t_{2}, u\right)+I^{f}\left(t_{1}, t_{2}, u\right)-I^{f}\left(t_{1}, t_{2}, \tilde{u}\right) \\
& \quad \geq 3 \Delta / 2-\Delta / 16>0,
\end{aligned}
$$

a contradiction (see (2.1)). The obtained contradiction proves the proposition.

In the sequel we also need the next two propositions proved in [13].

Proposition 2.8 ([13, Proposition 2.8]). Let $f \in \mathscr{M}$ and let $0<c_{1}<c_{2}<\infty$, $c_{3}, \epsilon>0$. Then there exists a neighborhood $V$ of $f$ in $\mathscr{M}$ such that for each $g \in V$, each $T_{1}, T_{2} \geq 0$ satisfying $T_{2}-T_{1} \in\left[c_{1}, c_{2}\right]$ and each $y, z \in \mathbb{R}^{n}$ satisfying $|y|,|z| \leq c_{3}$ the relation $\left|U^{f}\left(T_{1}, T_{2}, y, z\right)-U^{g}\left(T_{1}, T_{2}, y, z\right)\right| \leq \epsilon$ holds. 
PROPOSITION 2.9 ([13, Proposition 2.7]). Let $f \in \mathscr{M}, 0<c_{1}<c_{2}<\infty$ and $D, \epsilon>0$. Then there exists a neighborhood $V$ of $f$ in $\mathscr{M}$ such that for each $g \in V$, each $T_{1}, T_{2} \geq 0$ satisfying $T_{2}-T_{1} \in\left[c_{1}, c_{2}\right]$ and each a.c. function $x:\left[T_{1}, T_{2}\right] \rightarrow$ $\mathbb{R}^{n}$ satisfying $\min \left\{I^{f}\left(T_{1}, T_{2}, x\right), I^{g}\left(T_{1}, T_{2}, x\right)\right\} \leq D$ the relation $\mid I^{f}\left(T_{1}, T_{2}, x\right)-$ $I^{g}\left(T_{1}, T_{2}, x\right) \mid \check{\epsilon}$ holds.

\section{Proof of Proposition 1.2}

For each $h \in \mathscr{M}, \delta \in(0,1)$ and each $z \in \mathbb{R}^{n}$, let an a.c. function $Z_{\delta}^{h}:[0, \infty) \rightarrow \mathbb{R}^{n}$ be as guaranteed by Proposition 2.2.

Assume that $z \in \mathbb{R}^{n}, f \in \mathscr{M}$ and that for each $(t, x) \in[0, \infty) \times \mathbb{R}^{n}$ the function $f(t, x, \cdot): \mathbb{R}^{n} \rightarrow \mathbb{R}^{1}$ is convex.

For each integer $i \geq 0$, set

$$
z_{i}^{*}=Z_{\epsilon}^{f}(i) \quad \text { with } \quad \epsilon \in(0,1) .
$$

In view of Assertion (5) of Proposition 2.2, $z_{i}^{*}(i \geq 0)$ does not depend on $\epsilon$. By Proposition 2.1, there exists an a.c. function $Z^{*}:[0, \infty) \rightarrow \mathbb{R}^{n}$ such that for each integer $i \geq 0$,

$$
Z^{*}(i)=z_{i}^{*}, \quad I^{f}\left(i, i+1, Z^{*}\right)=U^{f}\left(i, i+1, Z^{*}(i), Z^{*}(i+1)\right) .
$$

It follows from (3.2), (3.1) and Assertion (4) of Proposition 2.2 that for each integer $k \geq 1$ and each $\epsilon \in(0,1)$

$$
\begin{aligned}
I^{f}\left(0, k, Z^{*}\right) & =\sum_{i=0}^{k-1} I^{f}\left(i, i+1, Z^{*}\right)=\sum_{i=0}^{k-1} U^{f}\left(i, i+1, z_{i}^{*}, z_{i+1}^{*}\right) \\
& =\sum_{i=0}^{k-1} U^{f}\left(i, i+1, Z_{\epsilon}^{f}(i), Z_{\epsilon}^{f}(i+1)\right) \leq I^{f}\left(0, k,, Z_{\epsilon}^{f}\right) \\
& \leq U^{f}\left(0, k, Z_{\epsilon}^{f}(0), Z_{\epsilon}^{f}(k)\right)+\epsilon=U^{f}\left(0, k, Z^{*}(0), Z^{*}(k)\right)+\epsilon .
\end{aligned}
$$

Since $\epsilon$ is an arbitrary element of $(0,1)$ we conclude that

$$
I^{f}\left(0, k, Z^{*}\right)=U^{f}\left(0, k, Z^{*}(0), Z^{*}(k)\right)
$$

for any integer $k \geq 0$. This implies that $I^{f}\left(0, T, Z^{*}\right)=U^{f}\left(0, T, Z^{*}(0), Z^{*}(T)\right)$ for any $T>0$. By Assertion (1) of Proposition 2.2 and Proposition 1.1 the function $Z^{*}$ is bounded and $(f)$-good. Proposition 1.2 is proved. 


\section{Overtaking optimal trajectories}

PROPOSITION 4.1. Let $f \in \mathscr{M}$ and property (P1) hold (see Theorem 1.3). Assume that $x:[0, \infty) \rightarrow \mathbb{R}^{n}$ is a bounded a.c. function such that for each $T>0$

$$
I^{f}(0, T, x)=U^{f}(0, T, x(0), x(T)) .
$$

Then $x$ is an $(f)$-overtaking optimal function.

Proof. By (4.1) and Proposition 1.1, $x$ is $(f)$-good. Assume that $x$ is not an $(f)$-overtaking optimal function. Then there is an a.c. function $y:[0, \infty) \rightarrow \mathbb{R}^{n}$ such that

$$
y(0)=x(0), \quad \limsup _{T \rightarrow \infty}\left[I^{f}(0, T, x)-I^{f}(0, T, y)\right] \geq 2 \epsilon
$$

with some positive number $\epsilon$. By Proposition 2.2, there is a bounded $(f)$-good function $Z:[0, \infty) \rightarrow \mathbb{R}^{n}$ such that $Z(0)=x(0)$ and that for each a.c. function $v:[0, \infty) \rightarrow \mathbb{R}^{n}$ either

$$
\lim _{T \rightarrow \infty}\left[I^{f}(0, T, v)-I^{f}(0, T, Z)\right]=\infty
$$

or

$$
\begin{array}{r}
\sup \left\{\left|I^{f}(0, T, v)-I^{f}(0, T, Z)\right|: T \in(0, \infty)\right\}<\infty, \\
\sup \{|v(t)|: t \in[0, \infty)\}<\infty .
\end{array}
$$

Since the function $x$ is $(f)$-good we conclude that

$$
\sup \left\{\left|I^{f}(0, T, x)-I^{f}(0, T, Z)\right|: T \in(0, \infty)\right\}<\infty .
$$

Relations (4.2) and (4.5) imply that (4.3) is not valid with $v=y$. Thus (4.4) is true with $v=y$. This implies that $y$ is a bounded $(f)$-good function. In view of property (P1)

$$
\lim _{t \rightarrow \infty}|x(t)-y(t)|=0
$$

Since $x, y$ are bounded functions we can choose a number

$$
\Delta>\sup \{|x(t)|+|y(t)|: t \in[0, \infty)\}+2 .
$$

In view of Proposition 2.3, there exists $\delta>0$ such that for each $T \geq 0$ and each $z_{i} \in \mathbb{R}^{n}, i=1, \ldots, 4$ satisfying

$$
\left|z_{i}\right| \leq \Delta, \quad i=1, \ldots, 4, \quad\left|z_{1}-z_{3}\right|,\left|z_{2}-z_{4}\right| \leq \delta
$$


the following inequality holds:

$$
\left|U^{f}\left(T, T+1, z_{1}, z_{2}\right)-U^{f}\left(T, T+1, z_{3}, z_{4}\right)\right| \leq \epsilon / 8 .
$$

It follows from (4.2) that there exists a sequence $\left\{T_{i}\right\}_{i=1}^{\infty} \subset(0, \infty)$ such that, for $i=1,2, \ldots$,

$$
T_{i+1} \geq T_{i}+8, \quad I^{f}\left(0, T_{i}, x\right)-I^{f}\left(0, T_{i}, y\right)>3 \epsilon / 2 .
$$

Equality (4.6) implies that there exists a natural number $j$ such that

$$
\left|x\left(T_{j}\right)-y\left(T_{j}\right)\right| \leq \delta .
$$

Consider an a.c. function $\tilde{x}:\left[0, T_{j}+1\right] \rightarrow \mathbb{B}^{n}$ such that

$$
\begin{gathered}
\tilde{x}(t)=y(t), \quad t \in\left[0, T_{j}\right], \quad \tilde{x}\left(T_{j+1}\right)=x\left(T_{j}+1\right), \\
I^{f}\left(T_{j}, T_{j}+1, \tilde{x}\right) \leq U^{f}\left(T_{j}, T_{j}+1, y\left(T_{j}\right), x\left(T_{j}+1\right)\right)+\epsilon / 8 .
\end{gathered}
$$

Relations (4.2) and (4.11) imply that

$$
\tilde{x}(0)=x(0), \quad \tilde{x}\left(T_{j}+1\right)=x\left(T_{j}+1\right) .
$$

It follows from (4.11) and (4.9) that

$$
\begin{aligned}
I^{f}\left(0, T_{j}\right. & +1, \tilde{x})-I^{f}\left(0, T_{j}+1, x\right) \\
= & I^{f}\left(0, T_{j}, \tilde{x}\right)+I^{f}\left(T_{j}, T_{j}+1, \tilde{x}\right) \\
& -I^{f}\left(0, T_{j}, x\right)-I^{f}\left(T_{j}, T_{j}+1, x\right) \\
\leq & I^{f}\left(0, T_{j}, y\right)-I^{f}\left(0, T_{j}, x\right)+U^{f}\left(T_{j}, T_{j}+1, y\left(T_{j}\right), x\left(T_{j}+1\right)\right) \\
& +\epsilon / 8-U^{f}\left(T_{j}, T_{j}+1, x\left(T_{j}\right), x\left(T_{j}+1\right)\right) \\
< & -3 \epsilon / 2+\epsilon / 8+U^{f}\left(T_{j}, T_{j}+1, y\left(T_{j}\right), x\left(T_{j}+1\right)\right) \\
& -U^{f}\left(T_{j}, T_{j}+1, x\left(T_{j}\right), x\left(T_{j}+1\right)\right) .
\end{aligned}
$$

By (4.7), (4.10) and the choice of $\delta($ see (4.8))

$$
\left|U^{f}\left(T_{j}, T_{j}+1, y\left(T_{j}\right), x\left(T_{j}+1\right)\right)-U^{f}\left(T_{j}, T_{j}+1, x\left(T_{j}\right), x\left(T_{j}+1\right)\right)\right| \leq \epsilon / 8 .
$$

Combined with (4.13) this inequality implies that

$$
I^{f}\left(0, T_{j}+1, \tilde{x}\right)-I^{f}\left(0, T_{j}+1, x\right)<-3 \epsilon / 2+\epsilon / 8+\epsilon / 8<0 .
$$

This contradicts (4.1). The contradiction we have reached proves the proposition.

Proposition 1.2 and Proposition 4.1 imply the following result. 
PROPOSITION 4.2. Assume that $f \in \mathscr{M}$, for each $(t, x) \in[0, \infty) \times \mathbb{R}^{n}$ the function $f(t, x, \cdot): \mathbb{R}^{n} \rightarrow \mathbb{R}^{1}$ is convex and that property ( $\mathrm{P} 1$ ) holds (see Theorem 1.3). Then for each $z \in \mathbb{R}^{n}$ there exists a bounded $(f)$-overtaking optimal function $Z:[0, \infty) \rightarrow$ $\mathbb{R}^{n}$ satisfying $Z(0)=z$.

Proposition 4.3. Let $f \in \mathscr{M}$ and assume that property (P1) holds (Theorem 1.3). Assume that $v_{1}, v_{2}:[0, \infty) \rightarrow \mathbb{R}^{n}$ are bounded a.c. functions, $v_{1}$ is $(f)$-overtaking optimal, $T_{0}>0$,

$$
v_{1}(t)=v_{2}(t), \quad t \in\left[0, T_{0}\right]
$$

and

$$
I^{f}\left(T_{0}, \tau, v_{2}\right)=U^{f}\left(T_{0}, \tau, v_{2}\left(T_{0}\right), v_{2}(\tau)\right) \text { for each } \tau>T_{0} .
$$

Then $v_{2}$ is an $(f)$-overtaking optimal function.

Proof. Clearly $v_{1}$ is an $(f)$-good function. We will show that $v_{2}$ is an $(f)$-good function. Choose a number

$$
M_{0}>\sup \left\{\left|v_{i}(t)\right|: t \in[0, \infty), i=1,2\right\} .
$$

By Proposition 2.6 there is $M_{1}>0$ such that

$$
M_{1}>\sup \left\{\begin{array}{l|l}
\left|U^{f}\left(t_{1}, t_{2}, y, z\right)\right| & \begin{array}{l}
t_{1} \geq 0, t_{2} \in\left[t_{1}+1 / 8, t_{1}+8\right] \\
y, z \in \mathbb{R}^{n},|y|,|z| \leq M_{0}+2
\end{array}
\end{array}\right\} .
$$

Let $\tau \geq T_{0}+2$. Consider an a.c. function $u:[0, \infty) \rightarrow \mathbb{R}^{n}$ such that

$$
\begin{gathered}
u(t)=v_{1}(t), \quad t \in[0, \tau-1], \quad u(\tau)=v_{2}(\tau), \\
I^{f}(\tau-1, \tau, u) \leq U^{f}\left(\tau-1, \tau, v_{1}(\tau-1), v_{2}(\tau)\right)+1 .
\end{gathered}
$$

Relations (4.18) and (4.14) imply that

$$
u\left(T_{0}\right)=v_{2}\left(T_{0}\right), \quad u(\tau)=v_{2}(\tau) .
$$

By (4.14), (4.15), (4.18) and (4.19),

$$
I^{f}(0, \tau, u)-I^{f}\left(0, \tau, v_{2}\right)=I^{f}\left(T_{0}, \tau, u\right)-I^{f}\left(T_{0}, \tau, v_{2}\right) \geq 0 .
$$

In view of (4.16)-(4.18),

$$
\begin{aligned}
I^{f}(0, \tau, u)-I^{f}\left(0, \tau, v_{1}\right)= & I^{f}(\tau-1, \tau, u)-I^{f}\left(\tau-1, \tau, v_{1}\right) \\
\leq & U^{f}\left(\tau-1, \tau, v_{1}(\tau-1), v_{2}(\tau)\right)+1 \\
& -U^{f}\left(\tau-1, \tau, v_{1}(\tau-1), v_{1}(\tau)\right) \\
\leq & 2 M_{1} .
\end{aligned}
$$


Combined with (4.20) this relation implies that

$$
I^{f}\left(0, \tau, v_{2}\right) \leq I^{f}(0, \tau, u) \leq I^{f}\left(0, \tau, v_{1}\right)+2 M_{1}
$$

and

$$
I^{f}\left(0, \tau, v_{2}\right) \leq I^{f}\left(0, \tau, v_{1}\right)+2 M_{1} \text { for any } \tau>T_{0}+2 .
$$

Since $v_{1}$ is $(f)$-good we conclude that $v_{2}$ is an $(f)$-good function. By property (P1)

$$
\lim _{t \rightarrow \infty}\left|v_{2}(t)-v_{1}(t)\right|=0 .
$$

Since $v_{1}$ is $(f)$-overtaking optimal we have

$$
\underset{T \rightarrow \infty}{\limsup }\left[I^{f}\left(0, T, v_{1}\right)-I^{f}\left(0, T, v_{2}\right)\right] \leq 0 .
$$

We show that

$$
\limsup _{T \rightarrow \infty}\left[I^{f}\left(0, T, v_{2}\right)-I^{f}\left(0, T, v_{1}\right)\right] \leq 0 .
$$

Let $\epsilon>0$. By Proposition 2.3 there is $\delta>0$ such that for each $t \geq 0$, each $y_{i}, z_{i} \in \mathbb{R}^{n}$, $i=1,2$, satisfying

$$
\left|y_{i}\right|,\left|z_{i}\right| \leq M_{0}+1, \quad i=1,2, \quad\left|y_{i}-z_{i}\right| \leq \delta, \quad i=1,2,
$$

the following inequality holds:

$$
\left|U^{f}\left(t, t+1, y_{1}, y_{2}\right)-U^{f}\left(t, t+1, z_{1}, z_{2}\right)\right| \leq \epsilon / 8 .
$$

In view of $(4.21)$, there is $T_{1}>T_{0}+4$ such that

$$
\left|v_{2}(t)-v_{1}(t)\right| \leq \delta \quad \text { for all } t \in\left[T_{1}, \infty\right) .
$$

Let $T>T_{1}$ and consider an a.c. function $w:[0, T+1] \rightarrow \mathbb{R}^{n}$ such that

$$
\begin{gathered}
w(t)=v_{1}(t), \quad t \in[0, T], \quad w(T+1)=v_{2}(T+1), \\
I^{f}(T, T+1, w) \leq U^{f}\left(T, T+1, v_{1}(T), v_{2}(T+1)\right)+\epsilon / 8 .
\end{gathered}
$$

Relations (4.14) and (4.27) imply that

$$
w(t)=v_{2}(t), \quad t \in\left[0, T_{0}\right], \quad w(T+1)=v_{2}(T+1) .
$$

By (4.15) and (4.28),

$$
\begin{aligned}
& I^{f}(0, T+1, w)-I^{f}\left(0, T+1, v_{2}\right) \\
& \quad=I^{f}\left(T_{0}, T+1, w\right)-I^{f}\left(T_{0}, T+1, v_{2}\right) \geq 0 .
\end{aligned}
$$


It follows from (4.16), (4.26), (4.27) and the choice of $\delta$ (see (4.24), (4.25)) that

$$
\begin{aligned}
I^{f}(0, T & +1, w)-I^{f}\left(0, T+1, v_{1}\right) \\
= & I^{f}(T, T+1, w)-I^{f}\left(T, T+1, v_{1}\right) \\
\leq & U^{f}\left(T, T+1, v_{1}(T), v_{2}(T+1)\right)+\epsilon / 8 \\
& -U^{f}\left(T, T+1, v_{1}(T), v_{1}(T+1)\right) \\
\leq & \epsilon / 8+\epsilon / 8=\epsilon / 4 .
\end{aligned}
$$

By (4.29)-(4.30), $I^{f}\left(0, T+1, v_{2}\right) \leq I^{f}(0, T+1, w) \leq I^{f}\left(0, T+1, v_{1}\right)+\epsilon / 4$ for any $T>T_{1}$. This implies (4.23). In view of (4.22) and (4.23),

$$
\lim _{T \rightarrow \infty}\left[I^{f}\left(0, T, v_{1}\right)-I^{f}\left(0, T, v_{2}\right)\right]=0 .
$$

Since $v_{1}$ is $(f)$-overtaking optimal we conclude that $v_{2}$ is $(f)$-overtaking optimal. The proposition is proved.

\section{5. (STP) implies (P1), (P2) and (P3)}

Assume that $f \in \mathscr{M}$, for each $(t, x) \in[0, \infty) \times \mathbb{R}^{n}$ the function $f(t, x, \cdot)$ : $\mathbb{R}^{n} \rightarrow \mathbb{R}^{1}$ is convex, the function $f$ has (STP) and that a bounded a.c. function $X_{f}:[0, \infty) \rightarrow \mathbb{R}^{n}$ is the turnpike of $f$. In [14, Section 4] we showed that properties $(\mathrm{P} 1)$ and $(\mathrm{P} 3)$ hold. Now we show that (P2) holds.

By Proposition 4.2 there exists an $(f)$-overtaking optimal function $v:[0, \infty) \rightarrow$ $\mathbb{R}^{n}$ such that $v(0)=X_{f}(0)$. Let $v:[0, \infty) \rightarrow \mathbb{R}^{n}$ be any $(f)$-overtaking optimal function satisfying $v(0)=X_{f}(0)$. In view of Proposition 2.2, $v$ is bounded. Then it follows from (STP) that $v(t)=X_{f}(t), t \in[0, \infty)$.

\section{Basic lemma}

Assume that $f \in \mathscr{M}$, for each $(t, x) \in[0, \infty) \times \mathbb{R}^{n}$ the function $f(t, x, \cdot)$ : $\mathbb{R}^{n} \rightarrow \mathbb{R}^{1}$ is convex, $X_{f}:[0, \infty) \rightarrow \mathbb{R}^{n}$ is a bounded a.c. function and assume that properties (P1), (P2) and (P3) hold. In [14, Lemma 5.1] we proved the following important lemma.

LEMMA 6.1. For each $\epsilon>0$, there exist $T_{0}>0, \delta_{0}>0$ such that the following property holds: If $T_{1} \geq T_{0}, T_{2} \geq T_{1}+1$ and if an a.c. function $u:\left[T_{1}, T_{2}\right] \rightarrow \mathbb{R}^{n}$ satisfies, for $i=1,2$,

$$
\left|u\left(T_{i}\right)-X_{f}\left(T_{i}\right)\right| \leq \delta_{0} \quad \text { and } \quad I^{f}\left(T_{1}, T_{2}, u\right) \leq U^{f}\left(T_{1}, T_{2}, u\left(T_{1}\right), u\left(T_{2}\right)\right)+\delta_{0},
$$

then $\left|u(t)-X_{f}(t)\right| \leq \epsilon$, for $t \in\left[T_{1}, T_{2}\right]$. 
Now we prove our basic lemma.

LEMMA 6.2 (Basic Lemma). Let $\epsilon>0$. Then there exists $\delta>0$ such that for each $T_{1} \geq 0, T_{2} \geq T_{1}+1$ and each a.c. function $v:\left[T_{1}, T_{2}\right] \rightarrow \mathbb{R}^{n}$ satisfying

(6.1) $\left|v\left(T_{i}\right)-X_{f}\left(T_{i}\right)\right| \leq \delta \quad$ and $\quad I^{f}\left(T_{1}, T_{2}, v\right) \leq U^{f}\left(T_{1}, T_{2}, v\left(T_{1}\right), v\left(T_{2}\right)\right)+\delta$, for $i=1,2$, the following inequality holds:

$$
\left|X_{f}(t)-v(t)\right| \leq \epsilon, \quad t \in\left[T_{1}, T_{2}\right]
$$

ProOF. By Lemma 6.1, there exist $\tau_{0}, \delta_{0} \in(0, \epsilon / 16)$ such that the following property holds:

(P4) If $T_{1} \geq \tau_{0}, T_{2} \geq T_{1}+1$ and an a.c. function $v:\left[T_{1}, T_{2}\right] \rightarrow \mathbb{R}^{n}$ satisfies $\left|v\left(T_{i}\right)-X_{f}\left(T_{i}\right)\right| \leq \delta_{0}, i=1,2$, and $I^{f}\left(T_{1}, T_{2}, v\right) \leq U^{f}\left(T_{1}, T_{2}, v\left(T_{1}\right), v\left(T_{2}\right)\right)+\delta_{0}$, then $\left|v(t)-X_{f}(t)\right| \leq \epsilon, t \in\left[T_{1}, T_{2}\right]$.

We may assume without loss of generality that $\delta_{0}<1$. Choose

$$
M_{0}>4+\sup \left\{\left|X_{f}(t)\right|: t \in[0, \infty)\right\}
$$

By Proposition 2.4 there exists a number $M_{1}>1$ such that for each $T_{1} \geq 0, T_{2} \geq$ $T_{1}+8^{-1}$ and each a.c. function $v:\left[T_{1}, T_{2}\right] \rightarrow \mathbb{R}^{n}$ satisfying $(i=1,2)$

$$
\left|v\left(T_{i}\right)\right| \leq M_{0}+4 \quad \text { and } \quad I^{f}\left(T_{1}, T_{2}, v\right) \leq U^{f}\left(T_{1}, T_{2}, v\left(T_{1}\right), v\left(T_{2}\right)\right)+4
$$

the following inequality holds:

$$
|v(t)| \leq M_{1}, \quad t \in\left[T_{1}, T_{2}\right]
$$

In view of property $(\mathrm{P} 3)$, there exist $\delta_{1} \in\left(0, \min \left\{1, \delta_{0}\right\}\right)$ and $L_{1}>0$ such that the following property holds:

(P5) For each $T \geq 0$ and each a.c. function $v:\left[T, T+L_{1}\right] \rightarrow \mathbb{R}^{n}$ which satisfies

$$
\begin{gathered}
|v(T)|,\left|v\left(T+L_{1}\right)\right| \leq M_{1}+4, \\
I^{f}\left(T, T+L_{1}, v\right) \leq U^{f}\left(T, T+L_{1}, v(T), v\left(T+L_{1}\right)\right)+\delta_{1},
\end{gathered}
$$

there is $\tau \in\left[T, T+L_{1}\right]$ for which

$$
\left|X_{f}(\tau)-v(\tau)\right| \leq \delta_{0}
$$


Consider a sequence $\left\{\delta_{i}\right\}_{i=1}^{\infty} \subset(0,1)$ such that

$$
\delta_{i}<2^{-1} \delta_{i-1}, \quad i=2,3, \ldots
$$

Assume that the lemma is wrong. Then for each natural number $i$ there exist $T_{i 1} \geq 0$, $T_{i 2} \geq T_{i 1}+1$, an a.c. function $v_{i}:\left[T_{i 1}, T_{i 2}\right] \rightarrow \mathbb{R}^{n}$ such that

$$
\begin{gathered}
\left|X_{f}\left(T_{i j}\right)-v_{i}\left(T_{i j}\right)\right| \leq \delta_{i}, \quad j=1,2, \\
I^{f}\left(T_{i 1}, T_{i 2}, v_{i}\right) \leq U^{f}\left(T_{i 1}, T_{i 2}, v_{i}\left(T_{i 1}\right), v_{i}\left(T_{i 2}\right)\right)+\delta_{i}
\end{gathered}
$$

and $t_{i} \in\left[T_{i 1}, T_{i 2}\right]$ for which

$$
\left|X_{f}\left(t_{i}\right)-v_{i}\left(t_{i}\right)\right|>\epsilon .
$$

Let $i$ be a natural number. It follows from property (P4), (6.9), (6.10), (6.8) and (6.5) that

$$
T_{i 1}<\tau_{0} .
$$

By (6.9), (6.8), (6.3) and the choice of $M_{1}$ (see (6.4), (6.5)),

$$
\left|v_{i}(T)\right| \leq M_{1}, \quad t \in\left[T_{i 1}, T_{i 2}\right] .
$$

We show that $t_{i} \leq \tau_{0}+L_{1}+2$. Assume the contrary. Then

$$
t_{i}>\tau_{0}+L_{1}+2 .
$$

Consider the restriction of $v_{i}$ to the interval

$$
\left[t_{i}-L_{1}-1, t_{i}-1\right] \subset\left(\tau_{0}+1, \infty\right) .
$$

Property (P5), (6.14), (6.12), (6.9) and (6.8) imply that there is

$$
\hat{t} \in\left[t_{i}-L_{1}-1, t_{i}-1\right]
$$

such that

$$
\left|X_{f}(\hat{t})-v_{i}(\hat{t})\right| \leq \delta_{0}
$$

By (6.15) and (6.13), $\hat{t}>\tau_{0}+1, T_{i 2}-\hat{t} \geq 1$. It follows from these inequalities, (6.16), (6.9), (6.8) and property (P4) that $\left|v_{i}(t)-X_{f}(t)\right| \leq \epsilon, t \in\left[\hat{t}, T_{i 2}\right]$. Combined with (6.15) this inequality implies that $\left|v_{i}\left(t_{i}\right)-X_{f}\left(t_{i}\right)\right| \leq \epsilon$, a contradiction. The contradiction we have reached proves that

$$
t_{i} \leq \tau_{0}+L_{1}+2
$$


Extracting if it is necessary a subsequence and re-indexing we may assume without loss of generality that there exist

$$
\begin{gathered}
\tilde{T}_{1}=\lim _{i \rightarrow \infty} T_{i 1} \in\left[0, \tau_{0}\right], \quad \tilde{t}=\lim _{i \rightarrow \infty} t_{i} \in\left[\tilde{T}_{1}, \tau_{0}+L_{1}+2\right], \\
\tilde{T}_{2}=\lim _{i \rightarrow \infty} T_{i 2} \in[\tilde{t}, \infty]
\end{gathered}
$$

(see (6.11), (6.17)).

It follows from (A.ii), (6.9), (6.12) and Proposition 2.6 that for each $\tau_{1} \in\left(\tilde{T}_{1}, \tilde{T}_{2}\right)$, $\tau_{2} \in\left(\tau_{1}, \tilde{T}_{2}\right)$ the sequence $\left\{I^{f}\left(\tau_{1}, \tau_{2}, v_{i}\right)\right\}_{i=1}^{\infty}$ is bounded.

By lower semicontinuity results [1], we may assume that there exists a function $\hat{v}:\left(\tilde{T}_{1}, \tilde{T}_{2}\right) \rightarrow \mathbb{R}^{n}$ such that the following property holds:

(P6) For each $\tau_{1} \in\left(\tilde{T}_{1}, \tilde{T}_{2}\right), \tau_{2} \in\left(\tau_{1}, \tilde{T}_{2}\right)$, the function $\hat{v}$ is a.c. on $\left[\tau_{1}, \tau_{2}\right]$, $v_{i}(t) \rightarrow \hat{v}(t)$ as $i \rightarrow \infty$ uniformly in $t \in\left[\tau_{1}, \tau_{2}\right], v_{i}^{\prime} \rightarrow \hat{v}^{\prime}$ as $i \rightarrow \infty$ weakly in $L^{1}\left(\left[\tau_{1}, \tau_{2}\right] ; \mathbb{R}^{n}\right)$, and $I^{f}\left(\tau_{1}, \tau_{2}, \hat{v}\right) \leq \liminf _{i \rightarrow \infty} I^{f}\left(\tau_{1}, \tau_{2}, v_{i}\right)$.

We show that $X_{f}\left(\tilde{T}_{1}\right)=\lim _{t \rightarrow \tilde{T}_{1}^{+}} \hat{v}(t)$. Let $\Delta>0$. By Proposition 2.5, Proposition 2.6, (6.9) and (6.12) there is $\gamma \in(0,1 / 8)$ such that the following properties hold: For each integer $i \geq 1$ and each $t_{1}, t_{2} \in\left[T_{i 1}, T_{i 2}\right]$ satisfying $\left|t_{1}-t_{2}\right| \leq 4 \gamma$ we have

$$
\left|v_{i}\left(t_{1}\right)-v_{i}\left(t_{2}\right)\right| \leq \Delta \text {. }
$$

For each $t_{1}, t_{2} \in[0, \infty)$ satisfying $\left|t_{1}-t_{2}\right| \leq 4 \gamma$, we have

$$
\left|X_{f}\left(t_{1}\right)-X_{f}\left(t_{2}\right)\right| \leq \Delta \text {. }
$$

Let $\tau \in\left(\tilde{T}_{1}, \tilde{T}_{1}+\gamma\right)$. Then for all sufficiently large natural numbers $i$

$$
T_{i 1}<\tau<\tilde{T}_{1}+\gamma<T_{i 1}+2 \gamma
$$

and in view of the choice of $\gamma$

$$
\left|v_{i}(\tau)-v_{i}\left(T_{i 1}\right)\right| \leq \Delta .
$$

It follows from (6.21), (6.22) and (6.9) that for all sufficiently large natural numbers $i$

$$
\left|v_{i}(\tau)-X_{f}\left(T_{i 1}\right)\right| \leq\left|v_{i}(\tau)-v_{i}\left(T_{i 1}\right)\right|+\left|v_{i}\left(T_{i 1}\right)-X_{f}\left(T_{i 1}\right)\right| \leq \Delta+\delta_{i} .
$$

By the choice of $\gamma,(6.20)$ and (6.18) for all sufficiently large natural numbers $i$,

$$
\left|X_{f}\left(\tilde{T}_{1}\right)-X_{f}\left(T_{i 1}\right)\right| \leq \Delta
$$

Combined with (6.23) this inequality implies that for all sufficiently large natural numbers $i$

$$
\left|v_{i}(\tau)-X_{f}\left(\tilde{T}_{1}\right)\right| \leq \mid v_{i}(\tau)-X_{f}\left(T_{i 1}|+| X_{f}\left(T_{i 1}\right)-X_{f}\left(\tilde{T}_{1}\right) \mid \leq \Delta+\delta_{i}+\Delta .\right.
$$


Thus by (P6) and (6.8)

$$
\left|\hat{v}(\tau)-X_{f}\left(\tilde{T}_{1}\right)\right|=\lim _{i \rightarrow \infty}\left|v_{i}(\tau)-X_{f}\left(\tilde{T}_{1}\right)\right| \leq \lim _{i \rightarrow \infty} 2 \Delta+\delta_{i}=2 \Delta .
$$

We have shown that for each $\tau \in\left(\tilde{T}_{1}, \tilde{T}_{1}+\gamma\right),\left|\hat{v}(\tau)-X_{f}\left(\tilde{T}_{1}\right)\right| \leq 2 \Delta$. Since $\Delta$ is an arbitrary positive number we conclude that

$$
X_{f}\left(\tilde{T}_{1}\right)=\lim _{\tau \rightarrow \tilde{T}_{1}^{+}} \hat{v}(\tau)
$$

Analogously we can show that if $\tilde{T}_{2}<\infty$, then

$$
X_{f}\left(\tilde{T}_{2}\right)=\lim _{\tau \rightarrow \tilde{T}_{2}^{-}} \hat{v}(t)
$$

We set $\hat{v}\left(\tilde{T}_{1}\right)=X_{f}\left(\tilde{T}_{1}\right)$ and if $\tilde{T}_{2}<\infty$, then $\hat{v}\left(\tilde{T}_{2}\right)=X_{f}\left(\tilde{T}_{2}\right)$. It follows from (P6), (6.9), (6.8) and Proposition 2.3 that for each $S_{1}, S_{2} \in\left(\tilde{T}_{1}, \tilde{T}_{2}\right)$ satisfying $S_{1}<S_{2}$

$$
\begin{aligned}
I^{f}\left(S_{1}, S_{2}, \hat{v}\right) & \leq \liminf _{i \rightarrow \infty} I^{f}\left(S_{1}, S_{2}, v_{i}\right) \\
& \leq \liminf _{i \rightarrow \infty}\left[U^{f}\left(S_{1}, S_{2}, v_{i}\left(S_{1}\right), v_{i}\left(S_{2}\right)\right)+\delta_{i}\right] \\
& =\liminf _{i \rightarrow \infty} U^{f}\left(S_{1}, S_{2}, v_{i}\left(S_{1}\right), v_{i}\left(S_{2}\right)\right)=U^{f}\left(S_{1}, S_{2}, \hat{v}\left(S_{1}\right), \hat{v}\left(S_{2}\right)\right)
\end{aligned}
$$

and

$$
I^{f}\left(S_{1}, S_{2}, \hat{v}\right)=U^{f}\left(S_{1}, S_{2}, \hat{v}\left(S_{1}\right), \hat{v}\left(S_{2}\right)\right) .
$$

By (P6), (6.12), (6.24) and (6.25), $\hat{v}$ is bounded. It follows from Proposition 2.7, (P6) and (6.24)-(6.26) that $\hat{v}$ is a.c. function on $\left[\tilde{T}_{1}, \tau\right)$ for each real $\tau \leq \tilde{T}_{2}$ and that the following properties hold:

$$
I^{f}\left(\tilde{T}_{1}, \tau, \hat{v}\right)=U^{f}\left(\tilde{T}_{1}, \tau, \hat{v}\left(T_{1}\right), \hat{v}(\tau)\right)
$$

for each $\tau \in\left(\tilde{T}_{1}, \tilde{T}_{2}\right]$ if $\tilde{T}_{2}<\infty$; and equality (6.27) holds for each $\tau>\tilde{T}_{1}$ if $\tilde{T}_{2}=\infty$.

We will show that $\hat{v}(\tilde{t}) \neq X_{f}(\tilde{t})$. It follows from Proposition 2.6 that there is $M_{2}>0$ such that

$$
\sup \left\{\begin{array}{l|l}
\left|U^{f}\left(s_{1}, s_{2}, x, y\right)\right| & \begin{array}{l}
s_{1} \geq 0, s_{2} \in\left[s_{1}+8^{-1}, s_{1}+8\right] \\
x, y \in \mathbb{R}^{n},|x|,|y| \leq M_{1}+2
\end{array}
\end{array}\right\}+4<M_{2} .
$$

Relations (6.28), (6.12) and (6.9) imply that the following property holds:

(P7) For each integer $i \geq 1$, each $s_{1}, s_{2} \in\left[T_{i 1}, T_{i 2}\right]$ satisfying $s_{2} \in\left[s_{1}+8^{-1}, s_{1}+8\right]$, $I^{f}\left(s_{1}, s_{2}, v_{i}\right)<M_{2}$. 
In view of property (P2), Proposition 2.6 and (6.3),

$$
\sup \left\{I^{f}\left(s_{1}, s_{2}, X_{f}\right): s_{1} \geq 0, s_{2} \in\left[s_{1}+8^{-1}, s_{1}+8\right]\right\}<\infty .
$$

By (P7), (6.29) and Proposition 2.5, there exists a positive number

$$
\gamma<\min \left\{1, \tilde{T}_{2}-\tilde{T}_{1}\right\} / 32
$$

such that the following properties hold:

(P8) For each $s_{1}, s_{2} \geq 0$ satisfying $\left|s_{1}-s_{2}\right| \leq \gamma,\left|X_{f}\left(s_{1}\right)-X_{f}\left(s_{2}\right)\right| \leq \epsilon / 64$.

(P9) For each integer $i \geq 1$, each $s_{1}, s_{2} \in\left[T_{i 1}, T_{i 2}\right]$ satisfying $\left|s_{1}-s_{2}\right| \leq \gamma$,

$$
\left|v_{i}\left(s_{1}\right)-v_{i}\left(s_{2}\right)\right| \leq \epsilon / 64 .
$$

Let $i$ be a natural number. We show that $t_{i}-T_{i 1}>\gamma$. Assume the contrary. Then $t_{i}-T_{i 1} \leq \gamma$ and by properties (P8) and (P9)

$$
\left|X_{f}\left(t_{i}\right)-X_{f}\left(T_{i 1}\right)\right|,\left|v_{i}\left(t_{i}\right)-v_{i}\left(T_{i 1}\right)\right| \leq \epsilon / 64 .
$$

Combining with (6.9), these inequalities imply that

$$
\begin{aligned}
\left|X_{f}\left(t_{i}\right)-v_{i}\left(t_{i}\right)\right| & \leq\left|X_{f}\left(t_{i}\right)-X_{f}\left(T_{i 1}\right)\right|+\left|X_{f}\left(T_{i 1}\right)-v_{i}\left(T_{i 1}\right)\right|+\left|v_{i}\left(T_{i 1}\right)-v_{i}\left(t_{i}\right)\right| \\
& \leq \epsilon / 64+\delta_{i}+\epsilon / 64 \leq \epsilon / 32+\delta_{0}<\epsilon / 32+\epsilon / 16 \\
& <\epsilon / 2
\end{aligned}
$$

This inequality contradicts $(6.10)$. The obtained contradiction proves that

$$
t_{i}-T_{i 1}>\gamma .
$$

Analogously we can show that

$$
T_{i 2}-t_{i}>\gamma .
$$

It follows from (6.30), (6.31), (6.17), (6.18) and property (P6) that

$$
\lim _{i \rightarrow \infty}\left|v_{i}\left(t_{i}\right)-\hat{v}\left(t_{i}\right)\right|=0 .
$$

Combined with (6.18) this equality implies that

$$
\lim _{i \rightarrow \infty} v_{i}\left(t_{i}\right)=\hat{v}(\tilde{t})
$$

By (6.18), $\lim _{i \rightarrow \infty} X_{f}\left(t_{i}\right)=X_{f}(\tilde{t})$. Combined with (6.32) and (6.10) this equality implies that

$$
\left|\hat{v}(\tilde{t})-X_{f}(\tilde{t})\right|=\lim _{i \rightarrow \infty}\left|v_{i}\left(t_{i}\right)-X_{f}\left(t_{i}\right)\right| \geq \epsilon
$$


Thus

$$
\hat{v}(\tilde{t}) \neq X_{f}(\tilde{t})
$$

There are two cases: (1) $\tilde{T}_{2}=\infty$; (2) $\tilde{T}_{2}<\infty$. Assume that $\tilde{T}_{2}<\infty$. Then (6.27) holds for each $\tau \in\left(\tilde{T}_{1}, \tilde{T}_{2}\right)$. By Proposition 2.7, $\hat{v}:\left[\tilde{T}_{1}, \tilde{T}_{2}\right] \rightarrow \mathbb{R}^{n}$ is an a.c. function and

$$
I^{f}\left(\tilde{T}_{1}, \tilde{T}_{2}, \hat{v}\right)=U^{f}\left(\tilde{T}_{1}, \tilde{T}_{2}, \hat{v}\left(\tilde{T}_{1}\right), \hat{v}\left(\tilde{T}_{2}\right)\right)
$$

We have $X_{f}\left(\tilde{T}_{i}\right)=\hat{v}\left(\tilde{T}_{i}\right), i=1,2$. Define an a.c. function $u:[0, \infty) \rightarrow \mathbb{R}^{n}$ by

$$
u(t)= \begin{cases}X_{f}(t), & t \in[0, \infty) \backslash\left(\tilde{T}_{1}, \tilde{T}_{2}\right), \\ \hat{v}(t), & t \in\left(T_{1}, T_{2}\right)\end{cases}
$$

Clearly $u$ is well defined. By property (P2) and (6.35), $\hat{v}$ is $(f)$-overtaking optimal. On the other hand, $u(0)=X_{f}(0)$ and $u(\tilde{t})=\hat{v}(\tilde{t}) \neq X_{f}(\tilde{t})$. This contradicts property (P2). Thus case (2) does not hold and $\tilde{T}_{2}=\infty$.

For each $t \geq 0$ satisfying $t<\tilde{T}_{1}$, set $\hat{v}(t)=X_{f}(t)$. Now (6.27) holds for each $\tau>\tilde{T}_{1}$. It follows from this fact, the boundedness of $\hat{v}$, the equality $\hat{v}\left(T_{1}\right)=X_{f}\left(\tilde{T}_{1}\right)$ and Proposition 4.3 that $\hat{v}$ is $(f)$-overtaking optimal. Now (6.34) contradicts property (P2). The obtained contradiction proves the lemma.

\section{Proof of Theorem 1.3}

In this section we prove the following theorem which is an extension of Theorem 1.3.

THEOREM 7.1. Let $f \in \mathscr{M}$, for each $(t, x) \in[0, \infty) \times \mathbb{R}^{n}$ the function $f(t, x, \cdot)$ : $\mathbb{R}^{n} \rightarrow \mathbb{R}^{1}$ is convex and let $X_{f}:[0, \infty) \rightarrow \mathbb{R}^{n}$ be a bounded a.c. function. Assume that properties $(\mathrm{P} 1)-(\mathrm{P} 3)$ from Theorem 1.3 hold.

Then for each $K, \epsilon>0$ there exist $\delta, L>0$ and a neighborhood $\mathscr{U}$ of $f$ in $\mathscr{M}$ such that the following property holds: For each $g \in \mathscr{U}$, each $T_{1} \geq 0, T_{2} \geq T_{1}+2 L$ and each a.c. function $v:\left[T_{1}, T_{2}\right] \rightarrow \mathbb{R}^{n}$ which satisfies

$$
\left|v\left(T_{1}\right)\right|,\left|v\left(T_{2}\right)\right| \leq K, I^{g}\left(T_{1}, T_{2}, v\right) \leq U^{g}\left(T_{1}, T_{2}, v\left(T_{1}\right), v\left(T_{2}\right)\right)+\delta,
$$

there exist $\tau_{1} \in\left[T_{1}, T_{1}+L\right], \tau_{2} \in\left[T_{2}-L, T_{2}\right]$ such that $\left|v(t)-X_{f}(t)\right| \leq \epsilon, t \in\left[\tau_{1}, \tau_{2}\right]$. Moreover, if $\left|v\left(T_{1}\right)-X_{f}\left(T_{1}\right)\right| \leq \delta$, then $\tau_{1}=T_{1}$, and if $\left|v\left(T_{2}\right)-X_{f}\left(T_{2}\right)\right| \leq \delta$, then $T_{2}=\tau_{2}$, 
PROOF. Let $K, \epsilon>0$. By Lemma 6.2 there exist $\delta_{0} \in(0,1)$ such that the following property holds:

(C1) If $T_{1} \geq 0, T_{2} \geq T_{1}+1$ and if an a.c. function $v:\left[T_{1}, T_{2}\right] \rightarrow \mathbb{R}^{n}$ satisfies

$\left|v\left(T_{i}\right)-X_{f}\left(T_{i}\right)\right| \leq \delta_{0}, \quad i=1,2, \quad I^{f}\left(T_{1}, T_{2}, v\right) \leq U^{f}\left(T_{1}, T_{2}, v\left(T_{1}\right), v\left(T_{2}\right)\right)+\delta_{0}$,

then $\left|v(t)-X_{f}(t)\right| \leq \epsilon, t \in\left[T_{1}, T_{2}\right]$.

By Proposition 2.4, there exist a number

$$
M_{0}>K+2+\sup \left\{\left|X_{f}(t)\right|: t \in[0, \infty)\right\}
$$

and a neighborhood $\mathscr{U}_{0}$ of $f$ in $\mathscr{M}$ such that the following property holds:

(C2) For each $g \in \mathscr{U}_{0}$, each $T_{1} \geq 0, T_{2} \geq T_{1}+1$ and each a.c. function $v$ : $\left[T_{1}, T_{2}\right] \rightarrow \mathbb{R}^{n}$ which satisfies

$$
\begin{aligned}
\left|v\left(T_{i}\right)\right| & \leq K+2+\sup \left\{\left|X_{f}(t)\right|: t \in[0, \infty)\right\}, \quad i=1,2, \\
I^{g}\left(T_{1}, T_{2}, v\right) & \leq U^{g}\left(T_{1}, T_{2}, v\left(T_{1}\right), v\left(T_{2}\right)\right)+4
\end{aligned}
$$

the inequality $|v(t)| \leq M_{0}$ holds for all $t \in\left[T_{1}, T_{2}\right]$.

In view of property (P3), there exist $\delta_{1} \in\left(0, \delta_{0}\right), L_{1}>0$ such that the following property holds:

(C3) For each $T \geq 0$ and each a.c. function $w:\left[T, T+L_{1}\right] \rightarrow \mathbb{R}^{n}$ which satisfies

$$
\begin{gathered}
|w(T)|,\left|w\left(T+L_{1}\right)\right| \leq M_{0}+4, \\
I^{f}\left(T, T+L_{1}, w\right) \leq U^{f}\left(T, T+L_{1}, w(T), w\left(T+L_{1}\right)\right)+\delta_{1},
\end{gathered}
$$

there is $\tau \in\left[T, T+L_{1}\right]$ for which $\left|X_{f}(\tau)-w(\tau)\right| \leq \delta_{0}$.

Proposition 2.8 implies that there exists a neighborhood $\mathscr{U}_{1}$ of $f$ in $\mathscr{M}$ such that the following property holds:

(C4) For each $T_{1} \geq 0, T_{2} \in\left[T_{1}+L_{1}, T_{1}+8\left(L_{1}+1\right)\right]$, each $g \in \mathscr{U}_{1}$, each $x, y \in \mathbb{R}^{n}$ satisfying $|x|,|y| \leq M_{0}+4,\left|U^{g}\left(T_{1}, T_{2}, x, y\right)-U^{f}\left(T_{1}, T_{2}, x, y\right)\right| \leq \delta_{1} / 32$.

By Proposition 2.6, there exists a number $M_{1}>0$ such that

$$
\sup \left\{\begin{array}{l|l}
\left|U^{f}\left(T_{1}, T_{2}, x, y\right)\right| & \begin{array}{l}
T_{1} \geq 0, T_{2} \in\left[T_{1}+1, T_{1}+8\left(L_{1}+1\right)\right], \\
x, y \in \mathbb{R}^{n},|x,| y \mid \leq M_{0}+4
\end{array}
\end{array}\right\} \leq M_{1} .
$$

It follows from Proposition 2.9 that there exists a neighborhood $\mathscr{U}_{2}$ of $f$ in $\mathscr{M}$ such that the following property holds:

(C5) For each $T_{1} \geq 0, T_{2} \in\left[T_{1}+L_{1}, T_{1}+8\left(L_{1}+1\right)\right]$, each $g \in \mathscr{U}_{2}$ and each a.c. function $v:\left[T_{1}, T_{2}\right] \rightarrow \mathbb{R}^{n}$ satisfying

$$
\min \left\{I^{f}\left(T_{1}, T_{2}, v\right), I^{g}\left(T_{1}, T_{2}, v\right)\right\} \leq M_{1}+8,
$$


the inequality $\left|I^{f}\left(T_{1}, T_{2}, v\right)-I^{g}\left(T_{1}, T_{2}, v\right)\right| \leq \delta_{1} / 32$ holds.

Define

$$
\mathscr{U}=\mathscr{U}_{0} \cap \mathscr{U}_{1} \cap \mathscr{U}_{2}
$$

choose a positive number $\delta<\min \left\{\epsilon, \delta_{0}, \delta_{1}\right\} / 32$ and set

$$
L=8+6 L_{1}
$$

Assume that $g \in \mathscr{U}, T_{1} \geq 0, T_{2} \geq T_{1}+2 L$ and an a.c. function $v:\left[T_{1}, T_{2}\right] \rightarrow \mathbb{R}^{n}$ satisfies $(i=1,2)$

$$
\left|v\left(T_{i}\right)\right| \leq K \quad \text { and } \quad I^{g}\left(T_{1}, T_{2}, v\right) \leq U^{g}\left(T_{1}, T_{2}, v\left(T_{1}\right), v\left(T_{2}\right)\right)+\delta
$$

By (7.6), (7.4) and property (C2),

$$
|v(t)| \leq M_{0}, \quad t \in\left[T_{1}, T_{2}\right]
$$

Let

$$
s_{1}, s_{2} \in\left[T_{1}, T_{2}\right], \quad s_{2}-s_{1} \in\left[L_{1}, 8\left(L_{1}+1\right)\right]
$$

It follows from (7.7), (7.4) and property (C4) that

$$
\left|U^{g}\left(s_{1}, s_{2}, v\left(s_{1}\right), v\left(s_{2}\right)\right)-U^{f}\left(s_{1}, s_{2}, v\left(s_{1}\right), v\left(s_{2}\right)\right)\right| \leq \delta_{1} / 32
$$

Relations (7.3), (7.7) and (7.8) imply that $U^{f}\left(s_{1}, s_{2}, v\left(s_{1}\right), v\left(s_{2}\right)\right) \leq M_{1}$. Combined with (7.9) this inequality implies that $U^{g}\left(s_{1}, s_{2}, v\left(s_{1}\right), v\left(s_{2}\right)\right) \leq M_{1}+\delta_{1} / 32$. In view of this inequality and (7.6),

$$
I^{g}\left(s_{1}, s_{2}, v\right) \leq U^{g}\left(s_{1}, s_{2}, v\left(s_{1}\right), v\left(s_{2}\right)\right)+\delta \leq M_{1}+\delta_{1} / 32+\delta
$$

By (7.10), (7.8), (7.4) and property (C5), $\left|I^{f}\left(s_{1}, s_{2}, v\right)-I^{g}\left(s_{1}, s_{2}, v\right)\right| \leq \delta_{1} / 32$. It follows from this inequality, (7.10), (7.9) and the choice of $\delta$ that

$$
\begin{aligned}
I^{f}\left(s_{1}, s_{2}, v\right) & \leq I^{g}\left(s_{1}, s_{2}, v\right)+\delta_{1} / 32 \leq U^{g}\left(s_{1}, s_{2}, v\left(s_{1}\right), v\left(s_{2}\right)\right)+\delta+\delta_{1} / 32 \\
& \leq U^{f}\left(s_{1}, s_{2}, v\left(s_{1}\right), v\left(s_{2}\right)\right)+\delta_{1} / 32+\delta+\delta_{1} / 32
\end{aligned}
$$

and

$$
I^{f}\left(s_{1}, s_{2}, v\right) \leq U^{f}\left(s_{1}, s_{2}, v\left(s_{1}\right), v\left(s_{2}\right)\right)+3 \delta_{1} / 32
$$

We have shown that the following property holds:

(C6) Inequality (7.11) is valid for each $s_{1}, s_{2}$ satisfying (7.8). 
Assume that

$$
\tau \in\left[T_{1}+L_{1}+1, T_{2}-L_{1}-1\right] .
$$

Relations (7.12) and (7.5) imply that $\tau-1-L_{1}, \tau+1+L_{1} \in\left[T_{1}, T_{2}\right]$. By property (C6)

$$
\begin{aligned}
& I^{f}\left(\tau-1-L_{1}, \tau-1, v\right) \\
& \quad \leq U^{f}\left(\tau-1-L_{1}, \tau-1, v\left(\tau-1-L_{1}\right), v(\tau-1)\right)+3 \delta_{1} / 32, \\
& I^{f}\left(\tau+1, \tau+1+L_{1}, v\right) \\
& \quad \leq U^{f}\left(\tau+1, \tau+1+L_{1}, v(\tau+1), v\left(\tau+1+L_{1}\right)\right)+3 \delta_{1} / 32 .
\end{aligned}
$$

It follows from (7.13), (7.14), (7.7) and property (C3) that there exist

$$
t_{1} \in\left[\tau-1-L_{1}, \tau-1\right], t_{2} \in\left[\tau+1, \tau+1+L_{1}\right]
$$

such that

$$
\left|X_{f}\left(t_{i}\right)-v\left(t_{i}\right)\right| \leq \delta_{0}, i=1,2 .
$$

Property (C6) implies that

$$
\begin{aligned}
& I^{f}\left(\tau-1-L_{1}, \tau+1+L_{1}, v\right) \\
& \quad \leq U^{f}\left(\tau-1-L_{1}, \tau+1+L_{1}, v\left(\tau-1-L_{1}\right), v\left(\tau+1+L_{1}\right)\right)+3 \delta_{1} / 32 .
\end{aligned}
$$

Together with (7.15) this inequality implies that

$$
I^{f}\left(t_{1}, t_{2}, v\right) \leq U^{f}\left(t_{1}, t_{2}, v\left(t_{1}\right), v\left(t_{2}\right)\right)+3 \delta_{1} / 32 .
$$

It follows from (7.15)-(7.17) and property (C1) that $\left|v(t)-X_{f}(t)\right| \leq \epsilon, t \in\left[t_{1}, t_{2}\right]$ and

$$
\left|v(\tau)-X_{f}(\tau)\right| \leq \epsilon
$$

We have shown that the following property holds:

(C7) Inequality (7.18) is true for each $\tau \in\left[T_{1}+L_{1}+1, T_{2}-L_{1}-1\right]$. (Note that $\left[T_{1}+L, T_{2}-L\right] \subset\left[T_{1}+L_{1}+1, T_{2}-L_{1}-1\right]$.)

Assume that

$$
\left|v\left(T_{1}\right)-X_{f}\left(T_{1}\right)\right| \leq \delta
$$

Let $\tau=T_{1}+L_{1}+1$. We have shown that there is

$$
t_{2} \in\left[T_{1}+L_{1}+1, T_{1}+L_{1}+1+1+L_{1}\right]
$$


such that

$$
\left|X_{f}\left(t_{2}\right)-v\left(t_{2}\right)\right| \leq \delta_{0}
$$

(see (7.15), (7.16)).

By property (C6),

$$
I^{f}\left(T_{1}, T_{1}+2 L_{1}+2, v\right) \leq U^{f}\left(T_{1}, T_{1}+2 L_{1}+2, v\left(T_{1}\right), v\left(T_{1}+2 L_{1}+2\right)\right)+3 \delta_{1} / 2 .
$$

Together with (7.20) this inequality implies that

$$
I^{f}\left(T_{1}, t_{2}, v\right) \leq U^{f}\left(T_{1}, t_{2}, v\left(T_{1}\right), v\left(t_{2}\right)\right)+3 \delta_{1} / 2 .
$$

It follows from (7.19)-(7.22) and property (C1) that $\left|v(t)-X_{f}(t)\right| \leq \epsilon, t \in\left[T_{1}, t_{2}\right]$ (note that $\left.\left[T_{1}, T_{1}+L_{1}+1\right] \subset\left[T_{1}, t_{2}\right]\right)$. Together with property (C7) this implies that (7.18) is true for each $\tau$ belonging to the interval $\left[T_{1}, T_{2}-L_{1}-1\right]$ which contains $\left[T_{1}, T_{2}-L\right]$.

Assume that

$$
\left|v\left(T_{2}\right)-X_{f}\left(T_{2}\right)\right| \leq \delta .
$$

Let $\tau=T_{2}-L_{1}-1$. We have shown (see (7.15), (7.16)) that there is

$$
t_{1} \in\left[T_{2}-L_{1}-2-L_{1}, T_{2}-L_{1}-2\right]
$$

such that

$$
\left|X_{v}\left(t_{1}\right)-v\left(t_{1}\right)\right| \leq \delta_{0}
$$

By (7.24) and Property (C6),

$$
I^{f}\left(t_{1}, T_{2}, v\right) \leq U^{f}\left(t_{1}, T_{2}, v\left(t_{1}\right), v\left(T_{2}\right)\right)+3 \delta_{1} / 2 .
$$

It follows from (7.23)-(7.26) and property (C1) that $\left|v(t)-X_{f}(t)\right| \leq \epsilon$ for any $t$ in the interval $\left[t_{1}, T_{2}\right]$ which contains $\left[T_{2}-L_{1}-2, T_{2}\right]$. Together with property (C7) this implies that (7.18) is true for each $\tau$ in the interval $\left[T_{1}+L_{1}+1, T_{2}\right]$ which contains $\left[T_{1}+L, T_{2}\right]$. This completes the proof of theorem.

\section{References}

[1] L. D. Berkovitz, 'Lower semicontinuity of integral functionals', Trans. Amer. Math. Soc. 192 (1974), 51-57. 
[2] Z. Dzalilov, A. F. Ivanov and A. M. Rubinov, 'Difference inclusions with delay of economic growth', Dynam. Systems Appl. 10 (2001), 283-293.

[3] Z. Dzalilov, A. M. Rubinov and P. E. Kloeden, 'Lyapunov sequences and a turnpike theorem without convexity', Set-Valued Analysis 6 (1998), 277-302.

[4] D. Gale, 'On optimal development in a multisector economy', Rev. Econom. Stud. 34 (1967), 1-19.

[5] V. L. Makarov, M. J. Levin and A. M. Rubinov, Mathematical economic theory: pure and mixed types of economic mechanisms (North-Holland, Amsterdam, 1995).

[6] V. L. Makarov and A. M. Rubinov, Mathematical theory of economic dynamics and equilibria (Nauka, Moscow, 1973); English translation (Springer, New York, 1977).

[7] M. A. Mamedov and S. Pehlivan, 'Statistical convergence of optimal paths', Math. Japon. 52 $(2000), 51-55$

[8] —, 'Statistical cluster points and turnpike theorem in nonconvex problems', J. Math. Anal. Appl. 256 (2001), 686-693.

[9] L. W. McKenzie, 'Turnpike theory', Econometrica 44 (1976), 841-866.

[10] R. Radner, 'Path of economic growth that are optimal with regard only to final states; a turnpike theorem', Rev. Econom. Stud. 28 (1961), 98-104.

[11] A. M. Rubinov, 'Economic dynamics', J. Soviet Math. 26 (1984), 1975-2012.

[12] P. A. Samuelson, 'A catenary turnpike theorem involving consumption and the golden rule', American Economic Review 55 (1965), 486-496.

[13] A. J. Zaslavski, 'Existence and uniform boundedness of approximate solutions of variational problems without convexity assumptions', Dynam. Systems Appl. 13 (2004), 161-178.

[14] - 'The structure of approximate solutions of variational problems without convexity', $J$. Math. Anal. Appl. 296 (2004), 578-593.

[15] - 'The turnpike property for approximate solutions of variational problems without convexity', Nonlinear Analysis 58 (2004), 547-569.

[16] _ 'Existence and uniform boundedness of optimal solutions of variational problems', Abstract and Appl. Analysis 3 (1998), 265-292.

[17] — 'The turnpike property for extremals of nonautonomous variational problems with vectorvalued functions', Nonlinear Analysis 42 (2000), 1465-1498.

[18] A. J. Zaslavski and A. Leizarowitz, 'Optimal solutions of linear control systems with nonperiodic integrands', Math. Oper. Res. 22 (1997), 726-746.

[19] - 'Optimal solutions of linear periodic control systems with convex integrands', Appl. Math. Optim. 37 (1998), 127-150.

\section{Department of Mathematics}

Technion-Israel Institute of Technology

32000, Haifa

Israel

e-mail: ajzasl@techunix.technion.ac.il 